\title{
CONTRACTION-FREE SEQUENT CALCULI FOR INTUITIONISTIC LOGIC: A CORRECTION
}

\author{
ROY DYCKHOFF
}

\begin{abstract}
We present a much-shortened proof of a major result (originally due to Vorob'ev) about intuitionistic propositional logic: in essence, a correction of our 1992 article, avoiding several unnecessary definitions.
\end{abstract}

$\S 1$. Introduction. One of the standard sequent calculi for Int (intuitionistic propositional logic) (with a language $\mathcal{L}$, based on atoms $P$ (i.e., atoms $p, q, r$ etc) using absurdity $\perp$, conjunction, disjunction and implication $\rightarrow$ ) is G3ip [4]. Sequents are of the form $\Gamma \Rightarrow A$ where $\Gamma$ is a multiset of formulae and $A$ is a formula. Combination of two multisets uses multiset sum. Its rules are

$$
\begin{aligned}
& \overline{\Gamma, P \Rightarrow P} \text { At } \quad \overline{\Gamma, \perp \Rightarrow E} L \perp \\
& \frac{\Gamma, A \Rightarrow B}{\Gamma \Rightarrow A \rightarrow B} R \rightarrow \quad \frac{\Gamma, A \rightarrow B \Rightarrow A \quad \Gamma, B \Rightarrow E}{\Gamma, A \rightarrow B \Rightarrow E} L \rightarrow \\
& \frac{\Gamma \Rightarrow A \quad \Gamma \Rightarrow B}{\Gamma \Rightarrow A \wedge B} R \wedge \quad \frac{\Gamma, A, B \Rightarrow E}{\Gamma, A \wedge B \Rightarrow E} L \wedge \\
& \frac{\Gamma \Rightarrow A_{i}}{\Gamma \Rightarrow A_{0} \vee A_{1}} R \vee \quad \frac{\Gamma, A \Rightarrow E \quad \Gamma, B \Rightarrow E}{\Gamma, A \vee B \Rightarrow E} L \vee .
\end{aligned}
$$

Note that (unless a loop-checker is added) root-first proof search in G3ip is nonterminating, because of the $L \rightarrow$ rule; so various authors proposed the trick of replacement of the $L \rightarrow$ rule by

$$
\begin{gathered}
\frac{\Gamma, P, B \Rightarrow E}{\Gamma, P, P \rightarrow B \Rightarrow E} L 0 \rightarrow \quad \frac{\Gamma, D \rightarrow B \Rightarrow C \rightarrow D \quad \Gamma, B \Rightarrow E}{\Gamma,(C \rightarrow D) \rightarrow B \Rightarrow E} L \rightarrow \\
\frac{\Gamma, C \rightarrow(D \rightarrow B) \Rightarrow E}{\Gamma,(C \wedge D) \rightarrow B \Rightarrow E} L \wedge \rightarrow \quad \frac{\Gamma, C \rightarrow B, D \rightarrow B \Rightarrow E}{\Gamma,(C \vee D) \rightarrow B \Rightarrow E} L \vee \rightarrow
\end{gathered}
$$

and thus we have the calculus G4ip for Int; it can be shown to be equivalent to G3ip. (Half of this is straightforward, using admissibility of Cut in G3ip.) Its crucial feature is that if formulae and then sequents are appropriately weighted, each rule's premisses are of weight less than the conclusion; so root-first proof search

Received April 18, 2018.

2010 Mathematics Subject Classification. 03F03, 03F50.

Key words and phrases. sequent calculus, intuitionistic propositional logic, termination.

(C) 2018, Association for Symbolic Logic 0022-4812/18/8304-0021 DOI: $10.1017 /$ js 1.2018 .38 
terminates. This both allows applications to uniform interpolation, as in (e.g.) [3] and provides an easily implemented calculus for a simple logic where backtracking is required (this may have some pedagogical value).

This article corrects the argument for equivalence given in [1], which is much too complicated - its author believes he understood it in about 1991, but struggles to convince himself. See [2] for references to other arguments.

§2. Routine results. Weakening (on the left) is easily shown to be admissible in the calculus G3ip. Routinely then, for any $\Gamma$ and $A$, the sequent $\Gamma, A \Rightarrow A$ is derivable. Contraction and $C u t$ are then shown to be admissible. This calculus then formalises the intuitionistic propositional logic Int - as axiomatised by the Hilbert system based on Modus Ponens and all axiom schemata (e.g., from Heyting's book) of Int. Checking this to be the case is routine.

Proposition 2.1. The rules $R \rightarrow, L \wedge, R \wedge, L \vee$ (and the second premiss of $L \rightarrow$ ) are invertible in G3ip.

Proof. Routine.

Proposition 2.2. The rules $L 0 \rightarrow, L \wedge \rightarrow, L \vee \rightarrow($ and the second premiss of $L \rightarrow$ ) of G4ip are invertible in G3ip.

Proof. By admissibility of Cut in G3ip.

We return to the question of equivalence between the two calculi. We recall from the Introduction that there is a definition of weight of sequents ensuring that rootfirst proof search terminates; crucially, this also allows use of induction on sequent weight, as illustrated below.

\section{$\S 3$. Main old result, with new proof.}

Theorem 3.1. Any sequent derivable in G3ip is derivable in G4ip.

Proof. By induction on the weight of the sequent. Without loss of generality, we may assume it is not of the form $\Gamma, P \Rightarrow P$ and its antecedent does not contain $\perp$. Using the invertibility results and the induction hypothesis, we may also assume that the succedent is not an implication or a conjunction, and that the antecedent contains no conjunction, disjunction, implication of the form $(C \wedge D) \rightarrow B$ or of the form $(C \vee D) \rightarrow B$, or pair of the form $P, P \rightarrow B$; in other words, it is irreducible.

Consider, among all derivations in G3ip of this sequent, one $\mathcal{D}$ with a shortest leftmost branch. (When computing this length for a $R \wedge$ or $L \vee$ step, the maximum of the lengths of the two premisses is used.) By the irreducibility, the last step must be by one of $R \vee$ or, with principal formula of the form $(C \rightarrow D) \rightarrow B$ or $P \rightarrow B$, the rule $L \rightarrow$; in the ultimate case, $P$ is not in the antecedent. We consider the possible cases in turn:

1. $R \vee$ is a rule common to the two calculi: the induction hypothesis deals with it.

2. $L \rightarrow$ with principal formula $(C \rightarrow D) \rightarrow B$ : so the final step of $\mathcal{D}$ has premisses $\Gamma,(C \rightarrow D) \rightarrow B \Rightarrow C \rightarrow D$ and $\Gamma, B \Rightarrow E$. We deal with the first premiss by using the invertibility (in G3ip) of $R \rightarrow$ (twice), the equivalence in G3ip (in the presence of $C)$ of $D \rightarrow B$ and $(C \rightarrow D) \rightarrow B$ and the induction hypothesis 
on the sequent $\Gamma, D \rightarrow B \Rightarrow C \rightarrow D$; then we deal with the second premiss by the induction hypothesis on $\Gamma, B \Rightarrow E$; then we use a $L \rightarrow$ step.

3. $L \rightarrow$ with principal formula $P \rightarrow B$ : so $\mathcal{D}$ is as follows:

$$
\frac{\begin{array}{c}
\mathcal{D}^{\prime} \\
P \rightarrow B, \Gamma^{\prime} \Rightarrow P \quad B, \Gamma^{\prime} \Rightarrow E
\end{array}}{P \rightarrow B, \Gamma^{\prime} \Rightarrow E} L \rightarrow .
$$

By irreducibility, $P \notin \Gamma^{\prime}$. So $\mathcal{D}^{\prime}$ must end in a step by a left rule (and by irreducibility that means the $L \rightarrow$ rule). If $P \rightarrow B$ is the principal formula then we can (by removing a step) shorten the leftmost branch of $\mathcal{D}$ and thus contradict the property of $\mathcal{D}$ of being a derivation of its end-sequent with shortest leftmost branch. So the principal formula must be a different implication; let it be $D \rightarrow F$, with $\Gamma^{\prime}=D \rightarrow F, \Gamma^{\prime \prime}$. Then $\mathcal{D}$ is

$$
\begin{aligned}
& \mathcal{D}_{0} \quad \mathcal{D}_{1}
\end{aligned}
$$

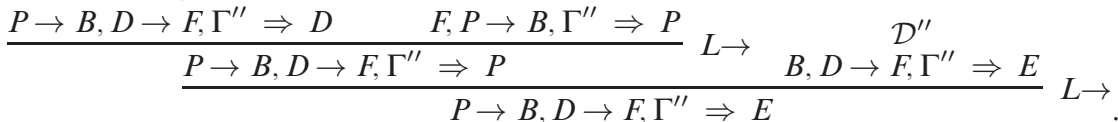

As in [1], using an inversion to obtain $\mathcal{D}_{2}$ from $\mathcal{D}^{\prime \prime}$, we can permute the proof $\mathcal{D}$ to obtain

$$
\frac{\mathcal{D}_{0}}{P \rightarrow B, D \rightarrow F, \Gamma^{\prime \prime} \Rightarrow D} \begin{array}{cc}
\mathcal{D}_{1} & \mathcal{D}_{2} \\
P \rightarrow B, D \rightarrow F, \Gamma^{\prime \prime} \Rightarrow E & \frac{P \rightarrow B, F, \Gamma^{\prime \prime} \Rightarrow P}{B, P \rightarrow B, \Gamma^{\prime \prime} \Rightarrow E}
\end{array} L \rightarrow
$$

which has a shorter leftmost branch than $\mathcal{D}$ and yet the same end-sequent. So this case cannot arise.

It follows that G4ip and G3ip are equivalent. This proof replaces that in Section 3 of [1]. It avoids complicated definitions of otherwise useless concepts like "awkward", "clumsy" and "sensible", and the uses of these definitions; it cuts the hard part of the proof from two pages to one; the proof no longer feels 'hard'; it gives a correct definition of "length" of proof. This may encourage wider extension to logics extending Int.

\section{REFERENCES}

[1] R. Dyckhoff, Contraction-free sequent calculi for intuitionistic logic, this JournaL, vol. 57 (1992), pp. 795-807.

[2] - Intuitionistic decision procedures since Gentzen, Advances in Proof Theory (R. Kahle, T. Strahm, and T. Studer, editors), Birkhäuser, Basel, 2016, pp. 245-267.

[3] A. PItTs, On an interpretation of second order quantification in first order intuitionistic propositional logic, this JournaL, vol. 57 (1992), pp. 33-52.

[4] A. S. Troelstra and H. Schwichtenberg, Basic Proof Theory, Cambridge University Press, Cambridge, 1996 and 2001.

\section{SCHOOL OF COMPUTER SCIENCE \\ UNIVERSITY OF ST ANDREWS \\ ST ANDREWS KY16 9AJ, UK}

